\title{
Il concetto di lunghezza e la retta.
}

\author{
(Di Rodolfo Bettazzi, a Torino.)
}

Il concetto di lunghezza si suole ordinariamente far dipendere da quelli di retta e di segmento: è scopo del presente articolo il dimostrare come possa stabilirsi anche indipendentemente da essi. Si verrà cosl a rendere legittima la nota definizione di segmento come "linea di minor lunghezza fra due " punti n la quale altrimenti, col ricorrere all'idea di lunghezza fondata su quella di retta, si aggira in un circolo vizioso.

亡̀ necessario avvertire che, per quello che segue, immagino già stabiliti con la dovuta esattezza i concetti di solido, di superficie, di linea, di punto con tutte le relative proprietà fondamentali, che io qui non credo dovere svolgere per non dilungarmi troppo e non eccedere in quello che non è strettamente argomento del presente lavoro. Per i concetti e le definizioni di solido, superficie ecc., rimando a quanto ho enunciato nel mio articolo: I postulati e gli enti geometrici $\left(^{*}\right)$ ed alla Nota $1 .^{\text {a }}$ in fine del presente lavoro; per le proprietà che di essi occorrerà applicare risparmierò ogni dimostrazione, indicando col segno (G) il momento in cui se ne farà uso. Se tali proprietà non enuncerò esplicitamente nel testo, citerò in apposite note in fine del lavoro quali esse siano, senza preoccuparmi se sono da ammettersi come postulati o da dimostrarsi come teoremi poichè ciò è indifferente per lo scopo che mi propongo, ma ritenendole come fatti geometrici indispensabili in ogni trattato di geometria.

Il numero notevole di postulati che introduco non rechi nessuna maraviglia, poichè è stata mia cura il cercare di enunciare i fatti (analoghi o corrispondenti a quelli della realtà) di cui si fa uso nelle dimostrazioni e dei quali spesso ci si serve inavvertentemente; essendo necessità che la geo-

(*) Periodico di Matematica per l'insegnamento secondario. Anno $1 .^{\circ}$ 
metria adopri ragionamenti nei quali neppure un'idea sia nascosta, come scienza logica in cui si devono solo dedurre conseguenze da premesse dopo avere enunciate queste tutte e chiaramente. Mi sono studiato di dimostrare il più che per me si poteva, persuaso della necessità di ridurre minimo il numero delle verità arbitrarie (postulati); ma quello che non ho saputo provare ho dato chiaramente come postulato, senza escludere con ciò che altri possa dimostrarlo. Del resto, vari dei miei postulati si trovano dati come tali anche in pregevolissime opere, per es.: in quelle di geometria del Pascil $\left(^{*}\right)$ e del Peano (**).

Osservazione. - Alcune note che si vedranno a piè di pagina non sono necessario completamento del testo, ma ne costituiscono semplici e non indispensabili dilucidazioni: e non vi sono inserite, perchè si parla spesso in esse di enti non ancora definiti o non definiti mai nel presente scritto, ma ai quali si ricorre perchè il loro concetto, certamente noto al lettore supposto non digiuno di studi geometrici, faccia meglio afferrare e giudicare il metodo seguito in questo lavoro.

\section{$\S 1$. Le sfere e l'apertura delle linee.}

1. Definizione I. ${ }^{\mathrm{a}}$ - "Una linea si dirà tratto se ha due estremi; ramo « se ha un estremo solo; linea chiusa se, essendo illimitata, si può spezzare in "due tratti; linea aperta se, essendo illimitata, si può spezzare in due rami."

Parlando in quello che segue di linee senz' altro aggiungere, intenderemo linee non intrecciate, che siano o tratti o linee chiuse.

Def. 2. ${ }^{a}$ - " Una superficie $\sigma$ che divide lo spazio in due parti si dirà "chiusa, quando l'una o l'altra di queste parti sia tale che in ciascun suo "punto posto l'estremo di un ramo qualunque $r$ e prese le infinite posizioni "di $r$, ciascuna di queste abbia punti anche nell'altra parte dello spazio. La " parte in quelle condizioni si dirà interna a $\sigma$, e si diranno interni a $\sigma \mathrm{i}$ "suoi punti."

2. Una coppia di punti dati è una figura: la mancanza di un ente che colleghi i due punti non toglie niente alla invariabilità di tale figura, e possiamo pensare che questa si muova comunque. Se di una coppia di punti

(") Pasch, Forlesungen über newere Geometrie. Leipzig, 188?.

(*) Peaxo, I principii di Geometria logicamente esposti, 'Torino, 1889. 
se ne tenga fermo uno, l'altro può assumere infinite posizioni: il seguente postulato fissa la natura dell' ente da esso generato.

Postulato $\boldsymbol{T}$. - Il luogo geometrico delle posizioni di un punto di una coppia il quale si muove mentre l'altro sta fermo, è una superficie chiusa a cui il punto immobile è interno.

Def. - "La superficie precedente si dice superficie sferica; il punto " fisso, centro; una qualunque delle posizioni delle coppie di punti dati, coppia "radiale; ogni tratto cogli estremi nei due punti di una coppia radiale, linea "radiale; quella delle due parti in cui è diviso lo spazio la quale contiene "il centro, sfera."

Cor. $10^{\circ}$ - Poichè una coppia di punti è rovesciabile (G) si ha che le due sfere originate da una coppia di punti prendendo per centro l'uno o l'altro di essi sono uguali.

Cor. $2 .^{\circ}$ - Il luogo geometrico delle posizioni di un estremo di un tratto, quando l'altro estremo è immobile ed il tratto assume tutte le posizioni possibili, è una superficie sferica di cui quella linea è radiale.

Cor. $3 .^{\circ}$ - Due superficie sferiche concentriche distinte non hanno punti comuni; quindi, date due superficie sferiche concentriche, ciascuna giace tutta da una parte dell' altra, (G) (*).

Cor. $4 .^{\circ}$ - I postulati ordinari sui solidi $(\mathbf{G})\left(^{* *}\right)$ ed il Corollario precedente ci permettono di concludere che se due sfere rese concentriche non coincidono, una è parte dell'altra. In tal caso, se $\boldsymbol{S}_{1}$ è la sfera parte di $\boldsymbol{S}_{2}$, deve la superficie $\sigma_{2}$ di $\boldsymbol{S}_{2}$ giacere rispetto a quella $\sigma_{1}$ di $\boldsymbol{S}_{1}$ da parte opposta del centro comune: altrimenti sarebbe $\sigma_{2}$ interna ad $\boldsymbol{S}_{1}$ e $\boldsymbol{\Phi}_{2}$ parte di $\mathbf{S}_{1}$, mentre invece è $\boldsymbol{S}_{1}$ parte di $\boldsymbol{S}_{2}(\mathbf{G})\left({ }^{* * *}\right)$.

Cor. $5 .^{\circ}$ - Date due sfere $\boldsymbol{S}_{1}$ ed $\boldsymbol{S}_{2}$ accade necessariamente uno ed uno solo dei tre casi: $\boldsymbol{S}_{1}$ uguale ad $\boldsymbol{\Phi}_{2}, \boldsymbol{S}_{1}$ parte di $\boldsymbol{S}_{2}, \boldsymbol{\Phi}_{2}$ parte di $\boldsymbol{\Phi}_{1}$.

Se dunque usiamo le parole "uguale" "maggiore" e " minore" nel senso consueto facendovi corrispondere i consueti segni $=,>,<$, può dirsi che date due sfere $\boldsymbol{S}_{1}$ ed $\boldsymbol{S}_{2}$, avviene necessariamente uno ed uno solo dei tre casi $\boldsymbol{S}_{1} \overline{\bar{\Sigma}} \boldsymbol{\Omega}_{2}$.

Cor. $60^{\circ}$ - Data una sfera, esistono sempre sfere maggiori e sfere minori di essa.

$\left({ }^{*}\right)$ Vedi Nota $2 .^{\text {a }}$ in fine di questo scritto.

(**) Vedi Nota $3 .^{a}$ in fine.

$\left(^{* *}\right)$ Vedi Nota $4 .^{a}$ in fine, 
3. Se dato un tratto $t$ si fa rotare attorno ad uno dei suoi estremi, esso genera coll'altro estremo una superficie sferica di cui è linea radiale $\left(2\right.$, Cor. $\left.2 .^{\circ}\right)$.

Def. I." - "La sfera descritta da un tratto come linea radiale si dirà "sfera di apertura del tratto."

Con. $10^{\circ}$ - La sfera di apertura di un tratto si ottiene indifferentemente tenendo fermo l'uno o l'altro estremo.

Def. 2. - " Data una linea chiusa diremo che essa ha una sfera di " apertura nulla: e questa sfera diremo uguale a quella delle altre linee " chiuse e minore di quelle di qualunque tratto.

Def. 3. - "Date due linee $l_{1}$ ed $l_{2}$ diremo che l'apertura di $l_{1}$ è uguale, " maggiore o minore di quella di $l_{2}$ secondochè la sfera di apertura di $l_{1} \grave{e}$ "uguale, maggiore o minore di quella di $l_{2}$."

Cor. $2 .^{\circ}$ - Le linee chiuse sono quelle di apertura minima.

Def. 4. ${ }^{a}$ - " Date infinite linee $\lambda$ ed un'altra $l$, diremo che le aperture a delle $\lambda$ possono differire da quelle di $l$ tanto poco quanto si vuole, quando " considerate le sfere di apertura delle $\lambda$ e di $l$, qualunque siano le sfere $\boldsymbol{S}_{1}$ " ed $\boldsymbol{S}_{2}$ prese una maggiore ed una minore di quella di apertura di $l$, esiste " sempre la sfera di apertura di qualche $\lambda$ che è minore di $\boldsymbol{S}_{1}$ e magg ore " di $\boldsymbol{\Phi}_{2}$. S'inteude che se $l$ è dj apertura nulla, la sfera da considerarsi è una "sola, la $\boldsymbol{S}_{i} \cdot n$

\section{$\S 2$. Sistemi chiusi di linee.}

4. Def. I. ${ }^{a}$ - "Diremo gruppo di linee una figura formata da un nu" mero finito di tratti $l_{1}, l_{2}, \ldots l_{n}$, tali che ciascuno abbia comune un estremo " col precedente ed uno col seguente, ed il primo non necessariamente con "l'ultimo, e che del resto non abbiano nessun altro punto comune fra loro."

Un gruppo di linee è una linea, che è un tratto se $l_{1}$ ed $l_{2}$ non hanno un estremo comune, ed una linea chiusa nel caso opposto $(\boldsymbol{G})\left({ }^{*}\right)$.

Def. 2. ${ }^{a}$ - " Data una linea, se per mezzo di punti la si scompone in un " numero finito di tratti e con questi si forma un gruppo qualunque (Def. $1 .{ }^{a}$ ), " questo gruppo è una linea che diremo linea di spezzamento della linea data."

Una linea ha infinite linee di spezzamento, sia per gli infiniti modi in

(*) Vedi Nota $5 .^{\text {a }}$ in fine. 
cui si può dividere in parti, sia per le infinite posizioni che nei gruppi si possono dare a queste parti.

Cor. - Se $l_{1}$ è linea di spezzamento di $l$, sarà $l$ linea di spezzamento di $l_{1}$.

5. Def. - "Diremo gruppo lineare di solidi il solido formato da un " numero finito di solidi $\boldsymbol{S}_{1}, \boldsymbol{S}_{2}, \ldots \boldsymbol{S}_{n}$, quando ciascuno di questi ha comune " una parte di superficie col precedente ed un'altra col seguente, ed il primo " e l'ultimo hanno o no parti di superficie comune, senza che $\mathrm{i}$ solidi abbiano, "oltre questi, nessun altro punto comune fra loro."

6. Def. I." - "Dato un sistema $\Sigma$ composto di una linea $l$ e di tutte " le sue linee di spezzamento, un'altra linea $l$ ' si dirà generata di $l$, quando "per ogni solido arbitrariamente scelto $\boldsymbol{S}$ sia possibile costruire un gruppo " lineare formato con solidi $\boldsymbol{S}_{1}, \boldsymbol{\Phi}_{2}, \ldots \boldsymbol{\Phi}_{n}$ uguali ad $\mathbf{S}$ od a sue parti, tale " che interne al solido così risultante possano disporsi $l^{\prime}$ ed una conveniente "linea $\lambda$ di $\Sigma$, in modo che: $10^{\circ}$ su ogni solido $\boldsymbol{S}_{r}$ non giaccia che un solo " tratto tanto di $l^{\prime}$ quanto di $\lambda ; 2 .^{\circ}$ le aperture di tutte queste $\lambda$ possano " differire da quella di $l$ tanto poco quanto si vuole (3, Def. $\left.4 .^{2}\right)$."

Def. 2. ${ }^{a}$ - "Data una linea $l$, se si costruiscono tutte le sue linee di " spezzamento e tutte le sue linee generate, si ottiene un altro sistema di linee "che si dirà sistema derivato di $l$ "."

Cor. $1 .^{\circ}-$ Se $l_{1}$ è linea di spezzamento di $l$, il sistema derivato di $l_{1}$ è lo stesso sistema derivato di $l$ (4, Cor.).

E opportuno completare questo corollario col

Postulato II. - Dato il sistema derivato di una linea $l$, ed una qualunque delle altre sue linee, $\lambda$ (sia questa di spezzamento di $l$ o generata), il sistema derivato di $\lambda$ è quello stesso di $l$.

Def. 3. ${ }^{a}$ - "Ogni sistema derivato di qualche linea si dirà sistema chiuso "di linee."

Cor. 2. ${ }^{\circ}$ - Se due sistemi chiusi di linee hanno una linea comune, essi sono identici (Post. II): e quindi due sistemi non identici non possono avere nessuna linea comune.

Cor. $3 .^{\circ}$ - Se in due sistemi chiusi di linee si ha che una linea dell' uno non è nell'altro, i due sistemi non possono avere linee comuni.

7. Def. I. ${ }^{a}$ - " Se le linee di un sistema chiuso si trasportano in modo " da avere tutte un estremo (se sono tratti) o un punto qualunque (se sono " chiuse) in uno stesso punto dato ad arbitrio $O$, si dirà che si ba il fascio "delle linee del sistema col centro $O$ : e l'insieme di tutte le sfere di aper- 
"tura in questa posizione (allora concentriche) si dirà il fascio delle sfere " stesse."

Cor. $10^{\circ}$ - Descritto il fascio delle sfere di apertura del sistema derivato di una linea $l$, se $l_{1}$ è una generata del sistema di $l$ e delle sue linee di spezzamento e si considera la sua sfera di apertura, fra due sfere qualunque concentriche ad essa, una maggiore ed una minore di essa, si trovarıo infinite sfere di apertura di linee di spezzamento di $l$, giacchè $l_{1}$ essendo loro generata le aperture di esse possono differire da quella di $l_{1}$ poco quanto si vuole $\left(6\right.$, Def. $\left.1^{a}\right)$.

- Dato il fascio delle sfere di apertura di un sistema, deve necessariamente accadere uno di questi due casi: o esiste una sfera concentrica ad esse (con tutte le infinite maggiori di essa) a cui nessuna delle sfere di apertura abbia punti esterni, o tale sfera non esiste.

Osservazione. - Vedremo in seguito (§ 5 ) che effettivamente può darsi l'un caso e l'altro.

Def. 2." - "Diremo sistemi finiti quei sistemi di linee chiusi tali che, " costruito il fascio delle loro sfere di apertura, esistono sfere concentriche ad " esse cui tutte le sfere di apertura sono interne: ed infiniti gli altri."

Postulato III. - Se l ̀̀ una linea di un sistema finito ed $l_{1} \grave{e}$ una sua parte, $l$ ed $l_{1}$ non fanno parte di uno stesso sistema $\left({ }^{*}\right)$ : e ciascuna linea del sistema derivato di $l_{1}$ è parte di convenienti linee del sistema derivato di l.

Cor. $2 .^{\circ}$ - Esistono infiniti sistemi chiusi non identici, per es.: quelli derivati di una linea $l$ di un sistema finito, di una sua parte qualunque $l_{1}$, di una parte $l_{2}$ di $l_{1}$ ecc.

Cor. $3 .^{\circ}$ - Una parte $l_{1}$ di una linea $l$ di un sistema finito non può essere uguale ad $l$, non potendo appartenere al suo sistema derivato.

Postulato IV. - Se $l_{1}$ ed $l_{2}$ sono tratti di sistemi finiti, sarà finito il sistema derivato di una linea che sia un gruppo qualunque di $l_{1}$ ed $l_{2}$.

CoR. $4 .^{\circ}$ - La proprietà vale anche per un numero qualunque (finito) di tratti.

Postulato V. - Dati due sistemi finiti non identici, in uno di essi esiste una linea $l_{1}$ parte di una $l_{2}$ dell'altro.

$\left(^{*}\right)$ Questa parte del postulato è destinata ad introdurre per' il concetto di lunghezza che si-sta svólgendo la proprietà che si suole sempre ammottere per gli enti finiti, quella che la parte non $\dot{0}$ equiralente al tutto. (Cfr. n. ${ }^{\circ}$, Cor. 2. ${ }^{\circ}$ ) 
Cor. 5. ${ }^{\circ}$ - Per il Postulato precedente ed il Post. III si conclude che dati due sistemi finiti non identici tutte le linee di uno di essi sono parti di convenienti linee dell'altro.

8. Def. 1. ${ }^{2}$ - "Data una classe $\Gamma$ di enti nella quale siano definiti $i$ " concetti di uguale, maggiore e minore, uno degli enti di essa, $\gamma$, si dirà " limite superiore di uno speciale sistema $\Gamma^{\prime}$ formato con alcuni di essi, o con " tutti, quando, appartenendo $\gamma$ o no a $\Gamma^{\prime}$, nessun ente di $\Gamma^{\prime}$ è maggiore di $\gamma$, " e per qualunque ente di $\Gamma$ minore di $\gamma$ ne esistono uno o più maggiori in $\Gamma^{\prime}$."

Postulato VI. - Per ogni sistema finito di linee esiste una sfera limite superiore di tulte le sfere di apertura delle linee del sistema, che $\grave{e}$ differente per sistemi differenti.

Def. 2. ${ }^{a}$ - " La sfera limite superiore delle sfere di apertura delle linee "di un sistema finito si dirà sfera limitatrice del sistema."

Con. $10^{\circ}$ - Se si considera il fascio delle sfere di apertura delle linee di un sistema finito e si dispone concentrica ad esso la sfera limitatrice, nessuna sfera di apertura ha punti esterni a questa, ma vi è qualche sfera di apertura che ha la superficie esterna ad una sfera concentrica alla sfera limitatrice e parte di essa, ma del resto qualunque.

Cor. 2.0 - Se la sfera limitatrice è una delle sfere di apertura del sistema, ogni linea del sistema che sia sua radiale ha l'apertura massima fra quelle del sistema.

Cor. $3 .^{\circ}$ - Sistemi di linee identici hanno la stessa sfera limitatrice e reciprocamente.

Teorema 1. - "Costruito un fascio delle linee di un sistema finito, u ciascuna in tutte le posizioni possibili, e posta col centro nel centro del "fascio la sfera limitatrice del sistema, tutte le linee del fascio hanno punti "non esterni a questa sfera."

Il teorema è chiaro per i punti estremi di queste linee, giacchè essi giacciono sulla superficie delle rispettive sfere di apertura, delle quali nessuna ha punti esterni alla sfera limitatrice $\boldsymbol{S}$. Se poi si considera un punto $P$ non estremo di una linea $l$, esso pure dev'essere interno ad $\boldsymbol{S}$. Infatti $P$, o da solo 0 col centro, divide $l$ in due parti $l_{1}$ ed $l_{2}$ che sono due tratti, uno $l_{1}$ cogli estremi $P$ ed $O$, un altro $l_{2}$ cogli stessi estremi se $l$ è chiusa o cogli estremi $P$ ed $O^{\prime}$ se $l$ è un tratto $O O$. Col centro in $P$ e colla linea radiale $l_{z}$ descrivo una superficie sferica $\left(2\right.$, Cor. $\left.2 .^{\circ}\right)$, la quale dovrà non aver punti 
esterni ad $\boldsymbol{S}$ : altrimenti, se $O^{\circ}$ fosse un punto di essa esterno ad $\mathbf{S}$, e $P O^{\prime}$ la corrispondente posizione della radiale $l_{2}$, il gruppo di $O P$ e $P O^{\prime \prime}$ sarebbe una linea di spezzamento di $l$, e quindi farebbe parte del sistema, talchè una sfera di apertura dovrebbe passare per $O^{\prime}$ ed essere quindi in parte esterna alla sfera limitatrice $\boldsymbol{S}$, contro il Corollario $10^{\circ}$. Avendosi che la superficie della sfera di centro $P$ e di radiale $l_{2}$ è non esterna a $\boldsymbol{S}$, saranno interni i suoi punti (G) e quindi il suo centro: e perciò $P$, punto scelto comunque su $l$, è interno, c, d. d.

Teorema $2 .^{\circ}$ - "Se $l$ è una linea di un sistema finito ed $l_{1} \dot{e}$ una " sua parte, anche il sistema derivato di $l_{1}$ è finito: e la sfera limitatrice del " secondo sistema è minore di quella del primo."

Infatti, se resi concentrici $\mathrm{i}$ fasci dei sistemi di $l$ ed $l_{1}$ un punto di una linea $l_{1}{ }_{1}$ del secondo fosse esterno alla sfera limitatrice $\mathbf{S}$ del primo, poichè (Post. III) $l^{\prime}{ }_{1}$ è parte di una linea conveniente $l^{\prime}$ del sistema di $l$, anche qualche linea di tal sistema avrebbe un punto esterno ad $\mathbf{\Phi}$ contro il Teor. $1 . \circ$ Tutte le sfere di apertura del sistema di $l_{1}$ sono dunque senza punti esterni ad $\boldsymbol{S}$, ed il sistema di $l_{1}$ è perciò finito $\left(7\right.$, Def. $\left.2 .^{a}\right)$ e la sua sfera limitatrice è $\boldsymbol{S}$ o una sfera minore $\left(8\right.$, Def. $\left.2 .^{\mathrm{R}}\right)$. E siccome tale sfera non può essere $\boldsymbol{S}$ a causa del Post. $V$, non essendo $l_{1}$ appartenente al sistema derivato di $l$, come sua parte (Post. III) sarà minore di $\mathbf{S}$, c. d. d.,

Con. $4 .^{\circ}$ - Se una linea $l$ di sistema finito è formata dal gruppo di due tratti $l_{1}$ ed $l_{2}$, sarà la sfera limitatrice di $l$ maggiore di quelle di $l_{1}$ ed $l_{2}$.

\section{$\S 3$. Le linee equivalenti e la lunghezza.}

9. Def. - "Due linee $l_{1}$ ed $l_{2}$ di sistemi finiti si dirà che sono equi"valenti o non equivalenti od anche che hanno uguale o disuguale lun"ghezza, secondochè appartengono o no ad uno stesso sistema chiuso."

Osservazione. - Per indicare i casi precedenti scriveremo rispettivamente $\bar{l}_{1}=\bar{l}_{2}, \bar{l}_{1}=\bar{l}_{2}$.

Cor. $10^{\circ}$ - Date due linee $l_{1}$ ed $l_{2}$ di sistemi finiti avviene necessariamente uno ed uno solo di questi due casi: o hanno uguale lunghezza, o l' hanno disuguale.

Cor. $20^{\circ}$ - Se $l$ è una linea di un sistema finito ed $l_{1}$ è una sua parte, si ha $\bar{l}=\bar{l}_{1}$ (Post. III). 
10. Def. - "Si dirà che la lunghezza di una linea $l$ è equivalente "alla somma delle lunghezze di altre due linee date $l_{1}$ ed $l_{2}$, quando, sup" posti finiti i sistemi derivati di $l$, di $l_{1}$ e di $l_{2}$, vi sia nel primo una linea " composta di due parti equivalenti ad $l_{1}$ ed $l_{2}$."

Osservazione. - Per indicare il caso precedente si scriverà $\bar{l}=\bar{l}_{1}+\bar{l}_{2}$.

Cor. - Date due linee $l_{1}$ ed $l_{2}$ di sistemi finiti, esistono infinite linee (tutte quelle di un sistema cbiuso) la cui lunghezza è equivalente alla somma delle lunghezze di $l_{1}$ ed $l_{2}$ : giacchè basta fare il sistema derivato della linea formata da un gruppo composto di $l_{1}$ ed $l_{2}$, o dei tratti loro parti (per il caso che $l_{1}$ ed $l_{2}$ siano linee chiuse) il quale è finito (Post. IV -7 , Cor. $4 .^{\circ}$ ).

11. Dati due sistemi finiti non identici, se $\boldsymbol{S}_{1}$ ed $\boldsymbol{S}_{2}$ sono le loro sfere limitatrici, avviene sempre uno ed uno solo di questi due casi: $\boldsymbol{S}_{1}$ [necessariamente (Post. VI) differente da $\boldsymbol{S}_{2}$ ] è parte di $\boldsymbol{S}_{2}, \quad$ o $\boldsymbol{S}_{\mathbf{2}}$ è parte di $\boldsymbol{S}_{1}$ (2, Cor. $\left.5 . .^{\circ}\right)$.

Def. - "Dati due sistemi chiusi di linee finiti e non identici, secon" dochè la sfera limitatrice del secondo è parte di quella del primo, o questa "dell'altra, diremo che le linee del primo sono prevalenti o suvvalenti a " quelle del secondo, oppure anche che le prime hanno lunghezza maggiore " o minore delle seconde.

"Ogni linea di sistema finito si dirà che ha minor lunghezza di ogni "linea di un sistema infinito."

Osservazione. - Per indicare $\mathrm{i}$ casi precedenti rispetto alle linee $l_{1}, l_{2}$ scriveremo $\overline{l_{1}}>\bar{l}_{2}, \bar{l}_{1}<\bar{l}_{2}$.

Con. $10^{0}$ - Date due linee $l_{1}, l_{2}$ di sistemi finiti avviene necessaria. mente uno ed uno solo dei tre casi $\bar{l}_{1}=\bar{l}_{2}, \bar{l}_{1}>\bar{l}_{2}, \overline{l_{1}}<\bar{l}_{2}$.

Cor. $2 .^{\circ}-$ Se $l_{1}$ è parte di $l$, sarà $\overline{l_{1}}<\bar{l}\left(8\right.$, Teor. $\left.2 . .^{\circ}\right)$.

Cor. $3 .^{\circ}$ - Data una linea di sistema finito ne esistono infinite altre di lunghezza minore della sua; per es.: le sue parti.

Cor. $4 .^{\circ}-$ Se $\bar{l}=\bar{l}_{1}+\bar{l}_{2}$, sarà $\bar{l}>l_{1}, \bar{l}>\bar{l}_{2}$, giacchè essendo $(10$, Def. $)$ una delle linee equivalenti ad $l$ composta di due parti equivalenti ad $l_{1}$ ed $l_{z}$, sarà la sfera limitatrice di $l$ maggiore di quelle di $l_{1}$ e di $l_{2}\left(8\right.$, Cor. $\left.4 .^{\circ}\right)$.

Cor. $5 .^{\circ}$ - Se una linea $l$ è prevalente ad un'altra $l_{1}$, sarà $l_{1}$ uguale ad una parte $l^{\prime}$ di una linea equivalente ad $l$. Ed infatti, i sistemi derivati di $l$ ed $l_{1}$ non essendo identici, saranno $\left(7\right.$, Cor. $\left.5 .^{\circ}\right)$ tutte le linee dell'uno parti di convenienti linee dell'altro. $\mathbf{E}$ non potendo essere quelle del sistema di $l$ parti di quelle dell'altro, altrimenti sarebbe (Cor. $\left.2 .^{\circ}\right) \vec{l}<\bar{l}_{1}$, contro l'jpo- 
tesi, dovranno essere quelle del sistema di $l_{\mathbf{1}}$ parti di quelle del sistema di $l$. In particolare sarà $l_{1}$ uguale ad una parte di una linea equivalente ad $l$.

12. Se $\bar{l}_{1}<\bar{l}$, ed è $l^{\prime}$ la linea equivalente ad $l$ della quale $l_{1}$ è parte $\left(11\right.$, Cor. $\left.5 .^{\circ}\right)$, indicando con $l_{1}^{\prime}$ uno qualunque dei gruppi ottenuti dalle parti che restano in $l^{\prime}$ toltovi $l_{1}$, sarà $\left(10\right.$, Def.) $\bar{l}=\bar{l}_{1}+\bar{l}_{1}$ e quindi (ivi) $\bar{l}=\bar{l}_{1}+\bar{l}_{1}$.

Def. - "Date due linee $l, l_{1}$ di cui $l_{1}$ sia suvvalente ad $l$, diremo che "la lunghezza di una linea $l_{1}$, è equivalente alla differenza fra quelle di $l$ "ed $l_{1}$, quando la lunghezza di $l$ sia la somma di quelle di $l_{1}$ ed $l_{1}$."

Osservazione. - Per indicare il caso precedente scriveremo $\bar{l}_{1}=\bar{l}-\bar{l}_{1}$.

Cor. - Date due linee $l$ ed $l_{1}$, tali che $\bar{l}_{1}<\bar{l}$, esistono sempre linee $l_{1}$ la cui lunghezza $\bar{l}_{1}$ è la differenza $\bar{l}-\bar{l}_{1}$ fra quelle di $l$ o di $l_{1}$.

13. Se ad ogni linea che appartenga a sistemi finiti si fa corrispondere un ente che si dica la sua lunghezza ed a questo- ente si attribuiscono le proprietà ora sviluppate per le linee corrispondenti riguardo ai concetti di equivalenza, prevalenza, suvvalenza, somma e differenza, si ha che le lunghezze delle linee appartenenti a sistemi finiti sono grandezze le quali costituiscono una classe rispetto all'operazione somma, classe che è ad una dimensione, ad un senso, illimitata e propria (*).

Il concetto di lunghezza è così pienamente stabilito.

\section{$\S 4$. Il segmento come linea di lunghezza minima.}

14. Passiamo a vedere come, introdotta la lunghezza, debba essa usarsi per dare del segmento una definizione rigorosa, nel senso già citato nell' introduzione.

Postulato VII. - Fra tutti $i$ tratti cogli stessi due estremi, ne esiste uno di un sistema finito ed uno solo che ha la lunghezza minima di fronte agli altri.

Def. 1. - "La linea di lunghezza minima fra tutte quelle cogli estremi "in due punti si dice segmento."

Def. 2. ${ }^{a}$ - " Nella sfera di apertura di un segmento, il segmento stesso, "che ne è linea radiale, si dice suo raggio."

(*) Vedi la mia Teoria delle Grandezze. Pisa, 1890, \$\$ 3, 16, 21, 28, 30, 31. 
Cor. $10^{\circ}$ - Un sistema di cui fa parte un segmento, cioè (Post. II) il sistema derivato di un segmento, è finito. L'esistenza di sistemi finiti è ora dunque una verità $(*)$.

Cor. $2 .^{\circ}$ - Una parte di un segmento non è uguale nè equivalente al segmento stesso $\left(7\right.$, Cor. $3 .^{\circ}-9$, Cor. $\left.2 .^{\circ}\right)$.

Teorema 1. ${ }^{\circ}$ - "Per due punti come estremi passa un segmento ed "uno solo."

E conseguenza immediata del Post. VII.

Osservazione. - Indicheremo il segmento di estremi $A$ e $B$ con $A B$.

Teorema 2. - "Se due segmenti sono raggi di una stessa sfera di "apertura sono uguali."

Infatti, se essi sono $O A, O B$, poichè la superficie della sfera di apertura è il luogo geometrico delle posizioni di un estremo quando l'altro estremo è fisso, sarà $B$ estremo di una posizione di $A$, e quindi per $O$ e $B$ passerà un segmento uguale ad $O A$; per il Teor. $10^{\circ}$ questo segmento è $O B$, e quindi $O A$ ed $O B$ sono uguali.

Cor. $3 .^{\circ}$ - Due segmenti disuguali hanno disuguale sfera di apertura.

15. Teorema $10^{\circ}$ - "Le due parti in cui un segmento $A B$ è di"viso $\left({ }^{* *}\right)$ da un punto $C$ che non ne sia un estremo, sono segmenti. "

Infatti, se per es.: la parte $C A$ non fosse un segmento, sostituendola col segmento $C A$, che è di minor lunghezza, insieme all' altra parte $C B$ darebbe un gruppo cogli estremi in $A$ e $B$ e di lunghezza minore di $A B$, il che non può essere.

(") Possiamo facilmento dare un esempio di un sistema infinito. Sopra una direzione $A B$ si stacchino a partire dall'estremo $A$ segmenti $A A_{1}, A A_{2}, A A_{3}, \ldots A A_{n}, \ldots$ di lunghezze rispettive $a, \frac{a}{2}, \frac{a}{3}, \ldots \frac{a}{n}, \ldots$; nei punti $A_{r}$ si inalzino segmenti $A_{r} A_{r}{ }_{r}$ perpendicolari ad $A B$, di lunghezza $\frac{a}{r}$, e si congiungano le coppie di punti $A^{\prime}{ }_{r}, A_{r+1}$. Risulta una linea $A_{1} A_{1}^{\prime} A_{2} A_{2}^{\prime} A_{3} A_{3}^{\prime}, \ldots$ composta di infiniti tratti, con un punto limite $A$, che fa da estremo, la quale quindi è da considerarsi come un tratto coi due estremi $A_{1}, A$. La linea ha lunghezza maggiore della somma dei segmenti $A_{r} A_{r}^{\prime}$, cioè maggiore di $\frac{a}{\mathrm{i}}+\frac{a}{2}+\frac{a}{3}+\ldots+\frac{a_{r}}{n}+\ldots$, e quindi non finita, giacchè la serie $\Sigma \frac{1}{n} \dot{\mathrm{e}}$ divergente. Il sistema derivato della linea in questione è chiaro che non é finito. Si puó discutere se figure come quelle qui usate siano da dirsi linee; ma mi pare che, comunque, almeno con un postulato speciale, si possa dar sempre loro questo nome.

$\left({ }^{*}\right)$ Vedi Nota $60^{a}$ in fine. 
Cor. $10^{\circ}$ - Se $B$ è un punto di un segmento $A C, C$ non è su $A B$ : giacchè altrimenti $A C$ sarebbe parte di $A B$, e quindi di $A C$, cioè $A C$ uguale ad una sua parte, contro il Cor. $2 .^{\circ}$ del n. ${ }^{\circ} 14$.

Teorema 2." - "Di due segmenti disuguali, uno (quello la cui sfera "di apertura è minore) è uguale ad una parte dell'altro."

Infatti si pongano $i$ segmenti dati $s_{1}$ ed $s_{2}$ con un estremo comune $O$, e se ne descrivano le sfere di apertura $\boldsymbol{S}_{1}$ ed $\boldsymbol{S}_{2}$ col centro in $O$. Se si ha $\boldsymbol{S}_{1}>\boldsymbol{S}_{2}$, la superficie di $\boldsymbol{S}_{1}$ è esterna ad $\boldsymbol{S}_{2}$, e quindi i suoi punti sono da parte opposta del centro rispetto alla superficie di $\boldsymbol{S}_{2}$. Se $P$ è uno di essi, il segmento $O P$, raggio di $\mathbf{s}_{1}$ e quindi uguale ad $s_{1}$, incontra la superficie di $\boldsymbol{S}_{2}$ almeno in un punto $(\mathbf{G})\left({ }^{*}\right)$ per es.: $C$, il quale divide $O P$ in due segmenti parti di $O P$ (Teor. 1. ${ }^{\circ}$ ). Una di esse, $O C$, sarà raggio di $\boldsymbol{S}_{2}$ e quindi uguale ad $s_{2}$ : si conclude che $s_{2}$ è uguale ad una parte di $s_{1}$.

Postulato VIIr. - Se due segmenti uguali hanno a comune un estremo ed un altro punto essi coincidono (**).

Teorema $30^{\circ}-$ "Se due segmenti $A C, A B$ con un estremo co" mune $A$ hanno comune un altro punto $B$, uno di essi ha il suo estremo " sull'altro (e quindi coincide o con esso o con una sua parte: 14 , Teor. $10^{\circ}-$ " 15 , Teor. $\left.1 .^{\circ}\right)$."

Infatti, condotte le due sfere di apertura col centro in $A$, o esse coincidono ed $\mathrm{j}$ scgmenti sono uguali (14, Teor. $2 .^{\circ}$ ) ed il Post. VIII dimostra il teorema - o non coincidono, e quella minore, sia per es.: quella di $A C$, incontrerà colla sua superficie il segmento $A D$ raggio dell' altra (che va ad un punto esterno ad essa) in un punto $D^{\prime}$, ed allora $A D^{\prime}$ ed $A C$ sono segmenti raggi di una stessa sfera di apertura e perciò uguali, che avendo $B$ comune, per quanto si è già detto, coincidono, talchè $C$ è un punto $D^{\prime}$ di $A D$. Cos̀̀ il teorema è dimostrato.

16. Teorema 1." - "Se un segmento $A B$ ha immobili gli estremi, "ha tutti i punti immobili."

Infatti, poichè intanto fra due punti passa un solo segmento, i punti di

(") Voli Nota $\sigma^{a}$ in tine.

(“) Questa appare veriti indipendente dal Post. VII o sue conseguenzo: giacché se the sermenti $A C, 1 D$ hanno un punto $B$ comune, il Post. VII conduce a concludere che il sermento $A B$, parte di $A C$ e di $A D$ e comune ad entrambi, ma nulla dice cil'ca le parti restanti $B C$ e $B D$. Anche il PAsch (loco eit.) ammotte qualcosa di simile nel suo Assioma VII, che corrisponde al mio T'eor. 3.. 
$A B$ possono al più scorrere sul segmento stesso: se dunque un punto $C$ di $A B$ si muova, dovrà andare su un altro punto $C^{\prime}$ di $A B$. Ora $C$ divide $A B$ nei segmenti $A C, C B\left(15\right.$, Teor. $\left.1 .^{\circ}\right)$ in uno dei quali, per es. in $A C$, dovrà essere $C^{\prime}$, talchè $A C^{\prime}$ è parte di $A C$. Ma nel moto le figure non si alterano, per cui $A C$, che si cambia in $A C^{\prime}$, dovrebbe essere uguale ad $A C^{\prime}$, contro i) Cor. $2 .^{\circ}$ del n. 14.

Teorema 2. - "Se un segmento $A B$ ha immobili due punti, anche "non estremi, $C$ e $D$, ha tutti i punti immobili."

Premettiamo che $C$ divide $A B$ in due parti, in una delle quali è $D:$ sia $D$ in $A C$. Se $A \in B$ movendosi vanno in $A^{\prime}$ e $B^{\prime}$, andrà $A C$ in $A^{\prime} C$, e $D$, che è di $A C$ ed è immobile, sarà anche di $A^{\prime} C$ : talchè $A C$ ed $A^{\prime} C$, avendo a comune un estremo $C$ ed un punto $D$, o saranno uno parte dell'altro, o coincideranno (15, Teor. $\left.3 .^{\circ}\right)$. Non accadendo il primo caso, perchè $A C$ ed $A^{\prime} C$ sono uguali essendo $A^{\prime}$ la nuova posizione di $A$, dorrà $A^{\prime}$ cadere in $A$. Allora sono uguali $A B^{\prime}$ ed $A B$, e, avendo, un punto comune $C$ (e $D$ ) oltre l'estremo $A$, per lo stesso motivo coincidono. Restando dunque $A^{\prime}$ in $A$ e $B^{\prime}$ in $B$, il segmento arrà immobili gli estremi, e quindi (Teor. $1^{\circ}$ ) tutti $\mathrm{i}$ punti.

17. Cor. $10^{\circ}$ - Due segmenti disuguali appartengono a sistemi chiusi disuguali (15, Teor. $2 .^{\circ}$ - Post. III) ed hanno quindi disuguale lunghezza.

Cor. 2. ${ }^{\circ}$ - Se di un sistema finito fa parte un segmento, non vi appartengono altri segmenti che $i$ suoi uguali (Cor. 1. ${ }^{\prime \prime}$ ): in altre parole due segmenti equivalenti sono uguali.

Teorema $10^{\circ}$ - "Se di un sistema chiuso fa parte un segmento, il a sistema, che è finito (14, Cor. 1."), ha per sfera limitatrice la sfera di aper"tura del segmento."

Se infatti si considera il fascio delle linee del sistema col centro in $O$, e $\sigma$ è la superficie della sfera $\mathbf{S}$ di apertura del segmento $O A$ del sistema, nessun punto di esse linee può cadere esterno ad $\mathbf{S}$. Giacchè se $P$ fosse un punto di una linea $l$ esterno ad $\mathbf{S}$, la parte $O P$ della linea (o la linea intera se $P$ è estremo, o una delle parti se la linea è chiusa) ha due punti da parti opposte di $\sigma$, e quindi incontra $\sigma$ almeno in un punto $B$. La parte $O B$ di $O P$ ha linghezza minore di $O P\left(11\right.$, Cor. $\left.2 .^{\circ}\right)$ e quindi di $l$, e percio il segmento $O B$ ha lunghezza minore di $l$. Ma $O B$ è una posizione di $O A$, ed $O A$ è del sistema di $l$, e quindi $\overline{O B}=\bar{l}$. Da questo assurdo discende che tutti $\mathrm{i}$ punti delle linee del sistema ed in particolare quelli delle sfere di apertura, sono non esterni ad $\mathbf{S}$. Poichè è una delle sfere di apertura, e tutte le altre sono interne ad essa, ò la sfera limitatrice. 
Cor. $30^{\circ}$ - Il segmento è fra le linee equivalenti quella di apertura massima $\left(8\right.$, Cor. $\left.2 .^{\circ}\right)$.

Teorema 2. - "Di ogni sistema finito fa parte un segmento."

Se infatti si costruisce la sua sfera limitatrice $\mathbf{S}$ di centro $O$, sia $O P$ un segmento suo raggio. Poichè $O P$ appartiene ad un sistema finito la cui sfera limitatrice è la sfera di apertura di $O P$ stesso (Teor. $1 .^{\circ}$ ) ed è quindi $\mathbf{s}$, il sistema dato e quello di $O P$ hanno la stessa sfera limitatrice, e quindi sono identici (Post. VI) talchè $O P$ fa parte del sistema dato, c. d. d.

Cor. $4 .^{\circ}$ - Sono sistemi finiti tutti e soli quelli di cui fa parte un segmento.

Cor. $5 .^{\circ}$ - Ogni linea di un sistema finito è equivalente ad un segmento e ad uno solo, che è quello del suo sistema, raggio della sua sfera limitatrice.

Def. I.a - "Ogni linea di un sistema finito si dirà rettificabile (a causa " della proprietà del Cor. 5..$^{\circ}$."

Def. 2. ${ }^{a}$ - "Il segmento equivalente ad una linea rettificabile può dirsi "lunghezza della linea."

Osservazione. - Colla definizione precedente le proprietà dell'ente lunghezza, dato come si è visto fin qui, relative ai concetti di grandezza, sono espresse dalle corrispondenti del segmento che lo rappresenta, come è facile a vedersi.

18. Def. - "Si dirà spezzata un gruppo di segmenti, e spezzata ina scritta in una linea $l$ una spezzata coi vertici (estremi dei segmenti) punti "di $l$, quando essa ed $l$ siano o entrambe senza estremi o cogli estremi co" muni, in modo che se $A, B$ sono due vertici consecutivi della spezzata, " su uno dei tratti (o sul tratto) $A B$ di $l$ non cadano altri vertici della "spezzata stessa."

Cor. $10^{\circ}$ - La lunghezza di una spezzata non chiusa che non sia un segmento, è maggiore di quella del segmento cogli stessi estremi.

Cor. $2 .^{\circ}$ - La lunghezza di una spezzata inscritta in una linea $l$ di sistema finito è minore di quella della linea $l$ : l'apertura è uguale.

19. Def. - "Diremo apertura di un tratto il segmento che ha i suoi "stessi estremi."

Osservazione. - Si conservano cosi evidentemente per l'apertura, come segmento, le proprietà espresse dalle definizioni già date di aperture uguali, maggiori, minori.

Cor. 1..$^{\circ}$ - Nel segmento, ed in esso solo, l'apertura è uguale alla lunghezza: ed è il segmento stesso. 
Cor. $2 .^{\circ}$ - In una linea rettificabile, che non sia il segmento, la lunghezza è maggiore dell'apertura, essendo questa un segmento minore di ogni linea coi suoi estremi, o essendo nulla.

Cor. $3 .^{\circ}$ - Dati più tratti per comporne un gruppo, fra tutti i modi possibili di disporli quello che dà una linea di maggiore apertura consiste nel disporre gli estremi dei tratti su uno stesso segmento: poichè in tal caso l'apertura è uguale alla lunghezza della spezzata inscritta nel gruppo coi vertici negli estremi dei tratti, mentre in ogni altro caso ne è minore (Cor. $2 .^{\circ}$ ).

\section{§ 5. La retta.}

20. Postulato IX. - "Esiste una linea aperta, tale che ogni suo "tratto `un segmento, e sulla quale esistono tratti uguali a qualunque seg"mento arbitrariamente dato."

Def. - "La linea precedente si dice retta."

Teorema 1. - "Per due punti qualunque passa una retta."

Infatti per $A, B$ come estremi passa un segmento, $A B$ : ora se $l$ è una retta, qualche suo tratto sarà uguale ad $A B$ (Post. IX) quindi si potrà muovere $l$ in modo che uno di essi coincida con $A B(\mathbf{G})\left(^{*}\right)$ e perciò passi per $A, B$. - Per $A$ e $B$ passa dunque $l$, c. d. d.

Teorema 2." - "Se due rette hanno a comune due punti coinci"dono, cioè per due punti passa una retta sola."

Infatti siano $l_{1}$ ed $l_{2}$ due rette passanti entrambi per due punti $A$ e $B$. Per $A$ e $B$ presi come estremi non passa che un segmento $A B$, comune quindi ad $l_{1}$ ed $l_{2}$, giacchè $\mathrm{i}$ tratti delle rette sono segmenti. Dico che anche gli altri punti di una delle linee sono della seconda e viceversa. Poichè infatti $(\mathbf{G})\left({ }^{* *}\right)$ i punti $A$ e $B$ dividono $l_{1}$ e così $l_{2}$, nel tratto (segmento) $A B$ e in due rami cogli estremi rispettivi $A$ e $B$, sia $P$ un punto del ramo di $l_{1}$ che ha per estremo $B$. Se con centro $A$ e raggio $A P$ si descrive la sfera, la sua superficie deve tagliare quel ramo di $l_{2}$ coll' estremo in $A$ che contiene $B$, giacchè la superficie della sfera è chiusa ed $A$ è un suo punto in-

(*) Vedi Nota 8. ${ }^{a}$ in fine.

(**) Vedi Nota $90^{a}$ in fine.

Amali di Matematica, tomo XX. 
terno e quindi questo ramo ha anche punti esterni e percio la incontra. Se $R$ è un punto d'incontro, $A R$ ¿̀ un segmento radiale della sfera, uguale quindi ad $A P\left(14\right.$, Teor..$\left.^{\circ}\right)$. Il punto $R$ non è su $A B$, altrimenti sarebbe $A R$ parte di $A B$ ed anche di $A P$, mentre è uguale ad $A P(14$, Cor. 2. $)$. I due segmenti $A P$ ed $A R$ sono uguali ed hanno comune l'estremo $A$ ed un punto $B$, quindi (Post. VIII) coincidono ed è $P$ in $R$, ossia $P$ è un punto $d i l_{2}$. Potendosi dimostrare che, reciprocamente, ogni punto di $l_{2}$, anche fuori di $A B$, è un punto di $l_{1}$, si conchiude che le due rette coincidono.

Cor. Due punti qualunque individuano una retta.

Teorema 3." - "Tutte le rette sono uguali."

Infatti se $A B$ è un tratto di una, $l$, e st un'altra $l^{\prime}$ cónsidero uno dei suoi uguali $A^{\prime} B^{\prime}$, trasporto $l$ in modo che $A B$ coincida con $A^{\prime} B^{\prime}(\mathbf{G})$. Allora $l$ ed $l^{\prime}$ avendo comuni due punti $A, A^{\prime}$ e $B, B^{\prime}$ coincidono (Teor. $2 .^{\circ}$ ) talchè sono uguali.

Teorema $4 .^{\circ}$ - "Se una retta ha due punti immobili, li ha immo"bili tutti."

Se infatti $A$ e $B$ sono questi punti e $C$ è un punto di $A B$, sarà $C$ immobile (16, Teor. $\left.10^{\circ}\right)$. Se $C$ è un punto qualunque non di $A B$, per es.: del ramo di $A$ che contiene $B$, il segmento $A C$ ha immobili i due punti $A$ e $B$ che esso contiene, e quindi li ha immobili tutti $\left(16\right.$, Teor. $\left.20^{\circ}\right)$; perciò $C$ è inmobile.

\section{§ 6. Coincidenza del concetto di lunghezza con quello ordinario.}

21. ¿̀ facile ora dimostrare che poichè la lunghezza di una linea rettificabile è uguale al segmento che le equivale (che è il segmento raggio della sfera limitatrice del sistema derivato della linea) il concetto di lunghezza così considerato coincide con l'ordinario concetto, quando questo si definisca dicendo lunghezza di una linea il segmento limite superiore (se esiste) della lunghezza di tutte le spezzate inscritte nella linea stessa. Si può cioè dimostrare che: $1 .^{\circ}$ data una linea rettificabile, nel senso usato in questo scritto, esiste il limite superiore della lunghezza di tutte le spezzate inseritte nella linea, ed è uguale al segmento equivalente, nel nostro senso, alla linea; $2 .^{\circ}$ data una linea per cui esiste il segmento limite superiore delle lunghezze delle spezzate inscritte, essa è rettificabile ed il segmento equivalente ad essa è uguale al limite superiore accennato. 
1. Se la linea $l$ è rettificabile ed è $s$ il segmento che le equivale, ogni sprezzata $s_{l}$ inscritta in essa ha lunghezza minore di quella di $l\left(18\right.$, Cor. $\left.2 .^{\circ}\right)$ : quindi si ha intanto che la lunghezza di $s$ è maggiore di quella di qualunque spezzata $s_{l}$. Per completare la dimostrazione basta provare $\left(8\right.$, Def. $\left.1 .^{\mathbf{a}}\right)$ che preso qualunque segmento $s^{\prime}$ minore di $s$ esistono sempre delle $s_{l}$ la cui lunghezza è mag'giore di $s^{\prime}$. Infatti, costruito il sistema derivato di $l$ e il fascio delle sue sfere di apertura, delle quali la sfera limitatrice $\boldsymbol{S}$ ha per raggio $s$, per il Cor. $1 .^{\circ}$ del n..$^{\circ} 8$ si ha che, presa la sfera concentrica di raggio $s^{\prime}$, fra le superficie di essa e di giacciono infinite superficie di sfere di apertura di linee del sistema, e quindi anche $\left(7\right.$, Cor. $\left.10^{\circ}\right)$ di linee di spezzamento di $l$. Presa una di tali linee di spezzamento, $l^{\prime}$, la sua apertura sarà dunque maggiore di $s^{\prime}$, e così dicasi di una qualunque spezzata inscritta in essa, la quale ha la stessa apertura: quindi la lunghezza di tale spezzata è $\left(19\right.$, Cor. $\left.2 .^{\circ}\right)$ maggiore di s'. Ma fra tali spezzate vi è quella i cui vertici cadono nei punti in cui si sono ricongiunte le parti nelle quali è stata divisa $l$ per comporre la linea di spezzamento $l$, e questa ha la stessa lunghezza della corrispondente inscritta in $l$ : talchè fra le spezzate inscritte in $l$ ve ne sono la cui lunghezza è maggiore di s'. Così è provato che $s$ è il limite superiore delle lunghezze delle spezzate inscritte in $l$, ed è dimostrata la prima parte del teorema.

2. Reciprocamente. Sia $l$ una linea per la qual'e si sappia esistente il limite superiore delle lunghezze delle spezzate inscritte. Si costruiscano tutte le sue linee di spezzamento; di esse quelle di apertura massima, fra le varie corrispondenti ad uno stesso modo di spezzare $l$, sono le linee in cui gli estremi delle parti ricongiunte sono in linea retta $\left(19\right.$, Cor. $\left.3 .^{\circ}\right)$, e per essa l'apertura è uguale al segmento equivalente alla spezzata inscritta in $l$ coi vertici nei punti di spezzamento. Se si considerano le aperture di tutte quelle speciali linee di spezzamento, si vengono a considerare $i$ segmenti equivalenti a tutte le spezzate possibili inscritte in $l$, e reciprocamente. Per le ipotesi fatte, esisterà dunque un limite superiore delle aperture di quelle speciali linee di spezzamento, e quindi anche di tutte le linee di spezzamento, essendo le prime quelle di apertura massima. Sia $s$ questo segmento: dopo aver descritto un fascio delle linee di spezzamento di $l$ ed una sfera $\$ \boldsymbol{S}$ concentrica al fascio e col raggio $s$, saranno tutte le sfere di apertura di quelle linee di spezzamento interne ad $\mathbf{S}$. Prese anche tutte le generate del sistema e condotte a far parte del fascio, nessun punto delle loro sfere di apertura sarà esterno ad $\mathbf{S}$; altrimenti poichè fra le superficie di esse e la superficie di $\boldsymbol{\Phi}$ non cadono sfere di apertura di 
linee di cui esse sono generate, si farebbe contro al Cor. $1 .^{\circ} \mathrm{del}$ n. ${ }^{\circ}$. Si conclude che esiste una sfera $\mathbf{S}$ a cui nessuna delle sfere di apertura del sistema derivato ha punti esterni; quindi un tale sistema è finito (7, Def. 2. $\left.{ }^{a}\right)$ ed $l$ è perciò rettificabile. Per la prima parte del teorema è allora il segmento equivalente ad $l$ uguale al limite superiore delle spezzate inscritte in $l$ ed è quindi il segmento $s$.

Il teorema è così dimostrato: ed è posto in chiaro come il concetto di lunghezza svolto in questo scritto non differisca da quello ordinario, quando almeno questo si definisca nel modo accennato in principio di questo paragrafo.

22. Il prof. Peano (*) definisce la lunghezza di una linea spezzando questa in parti, formando (sostituisco alle parole del prof. Penso il linguaggio che ho seguito in questo lavoro) tutti i gruppi di quelle parti e prendendo il limite superiore (quando esiste) delle aperture di questi gruppi. Poichè egli stesso dimostra che la lunghezza così definita coincide col limite superiore delle lunghezze delle spezzate inscritte, è chiaro che anche il suo concetto coincide col mio.

Del resto ciò si vede direttamente, osservando che il definire la lunghezza come fa $\mathrm{il}$ prof. Pesso equivale a chiamare lunghezza di una linea la linea equivalente alla data e che abbia l'apertura massima; e siccome tale linea i il segmento equivalente alla linea data, è chiaro che i due concetti si equivalgono.

\section{Appendice.}

23. Le considerazioni sin qui svolte mostrano un'altra, o forse non inopportuna, definizione del segmento.

Si supponga esposto quanto si ̀̀ detto nei primi tre capituli, cioè fino al n. 13 inclusivo.

Postulato VII'. - "In ogni sistema finito esiste una ed una sola "linea di apertura massima."

Def. - "Tale linea si dice segmento."

Con. 1." - Il segmento di un sistema ha per sfera di apertura la sfera limitatrice del sistema.

(*) Peano, Applicazioni geometriche del Calcolo infinitesimale. Tupino 1887, 19as. 161. 
Cor. $20^{\circ}-$ Se $s$ ed $s_{1}$ sono i segmenti di due diversi sistemi ed è $\bar{s}>\bar{s}_{1}$, sarà (11, Def.) la sfera limitatrice di $s$ maggiore di quella di $s_{1}$, e quindi (Cor. $1 .^{\circ}$ ) l'apertura di $s$ maggiore di quella di $s_{1}$.

Postulato VII". - "Per due punti come estremi passa sempre un "segmento."

Teorema. - "Il segmento cogli estremi in due punti è linea di mi- nima lunghezza fra quelle cogli stessi estremi. "

Infatti se $A$ e $B$ sono due punti, $s$ è il segmento $A B$ ed $l$ un'altra linea di sistema finito avente per estremi $A$ e $B$, avverrà uno ed uno solo dei tre casi: $\bar{s} \stackrel{\bar{l}}{\mathrm{l}}$. Non può essere $\bar{s}=\bar{l}$ avendo $s$ ed $l$ la stessa apertura, e dovendo invece il segmento (Def.) avere apertura maggiore delle linee equivalenti. Neppure può essere $\bar{s}>\bar{l}$, giacchè sarebbe ancora $\bar{s}>\bar{s}_{1}$, dove $s_{1}$ è il segmento equivalente ad $l$, e quindi $s$ arrebbe apertura maggiore di quella di $s_{1}$ (Cor. $\left.2^{\circ}\right)$ il quale a sua volta, perchè equivalente ad $l$, l'arrebbe per definizione maggiore di $l$ : e questo non può essere poichè $s$, che dovrebbe avere apertura maggiore di $l$, ha invece gli stessi estremi $A$ e $B$. Si conclude che $\bar{s}<\bar{l}$, c. d. d.

Osservazione. - Si potrebbe continuare a dimostrare le altre proprietà del segmento; ma ce ne dispensa questo teorema, che mostra l'identità dell'ente "segmento n definito colla primitiva maniera e coll'attuale.

\section{NOTE.}

Nota $1 .^{a}$ Stimo opportuno il citare qui brevemente e senza nessuna spiegazione i concetti e le definizioni fondamentali a cui intendo appoggiarmi, e che non si trovano completi nella mia Memoria citata "I postulati e gli enti geometrici ".

Le proposizioni che seguono sono postulati e definizioni. Alcune definizioni sono per brevità incluse nei postulati.

Post. - Esiste un ente che si dice spazio.

Post. - Esistono infiniti enti che non sono lo spazio, e si dicono parti dello spazio o solidi, e infiniti solidi che si dicono parti di un solido. 
Post. - Per ogni solido esistono uno o più enti (in numero finito) che non sono spazio nè solidi, il cui insieme costituisce il limite del solido, e ciascuno dei quali si dice superficie.

Per ogni superficie esistono infiniti enti, che si dicono sue parti, ed altri enti parti di queste, che pure sono parti della superficie data. Si dicono tutti superficie.

Post. - Per ogni superficie non limite di un solido esistono uno o più enti (in numero finito) non spazio, solidi o superficie il cui insieme è il limite o contorno della superficie, e che si dicono linee.

Per ogni linea esistono infiniti enti, sue parti, ed altri enti, parti di queste, che sono parti della linea data: si dicono tutte linee.

Post. - Per ogni linea non limite di una superficie esistono uno o due enti, che costituiscono il suo limite e ne sono estremi, ciascuno dei quali non ¿̀ spazio, nè solido, nè superficie, nè linea, e si dice punto.

Def. - Si dice che giacciono su un ente tutte le sue parti, i limiti di esso e di queste, e tutti gli enti che, per questa stessa definizione, giacciono su quelle parti o su quei limiti. Così su una linea giacciono infiniti punti, su una superficie infinite linee ed infiniti punti ecc.: e nello spazio giacciono tutti gli enti.

Def. - Se un ente $A$ giace su $B, A$ è di $B, B$ contiene $A$, passa per $A$.

Def. - Sono parti associate di un ente $A$ due parti di esso che, avendo comuni al più solo punti dei loro limiti, col loro insieme costituiscono l'ente $A$, cioè sono tali che i punti di $A$ sono tutti e soli quelli delle due parti.

Def. - Un ente $A$ di $B$ divide $B$, quando esso o solo o con parti associate del limite di $B$ costituisca il limite di due parti associate di $B$.

Def. - Un aggregato o gruppo di enti si dice figura.

Def. - Quando si considera una figura si dice anche che si hanno infinite figure coincidenti con essa.

Def. - Il gruppo di tutti i punti di una figura si dirà che forma la figura.

Post. - Un gruppo di punti forma una figura sola e le sue infinite coincidenti.

Post. - Esistono infiniti gruppi di punti che formano una stessa figura.

Def. - Una figura ha diverse posizioni quando è formata da diversi gruppi di punti. Quando si considera una figura con diverse posizioni, si dice che si hanno altrettante figure uguali.

Def. - Il passaggio della mente da una figura ad una sua uguale si dice moto della prima figura. 
Post. - Una figura può muoversi quando sono immobili uno o due dei suoi punti, ed assumere infinite posizioni.

Nota 2. ${ }^{a}$ (al Cor. 3..$^{\circ}$ del n. ${ }^{\circ}$ 2). - Se date due superficie $\sigma_{1}$ e $\sigma_{2}$, di cui $\sigma_{2}$ divide lo spazio, abbia $\sigma_{1}$ punti dalle due parti di $\sigma_{2}$, esse avranno anche punti comuni.

Nota 3. ${ }^{a}$ (al Cor. $4 .^{\circ}$ del $\mathrm{n}^{\circ}{ }^{2}$ ). - Se date due superficie chiuse $\sigma_{1}$ e $\sigma_{2}$ ciascuna giace tutta da una parte dell'altra, il solido interno ad una di esse è parte di quello interno all'altra.

Nota 4. ${ }^{a}$ (ivi). - I solidi interni a qualche superficie chiusa (finiti) sono tali che una loro parte non è uguale ad essi stessi.

Nota $5 .^{a}$ (alla Def. $1 .^{a}$ del n. $^{\circ} 4$ ). - Esiste una linea di cui quelle di un gruppo sono parti, e i cui punti sono tutti e soli quelli di queste parti.

Nota $6 .^{a}$ (al Teor. $10^{\circ}$ del n. ${ }^{\circ} 15$ ). - Un punto di un tratto divide questo in due parti.

Nota $7 .^{a}$ (al Teor. 2. ${ }^{\circ}$ del n.. 15). - Se una linea ha punti dalle due parti di una superficie che divide lo spazio, ha con essa punti comuni.

Nota $8 .^{a}$ (al Teor. $10^{\circ}$ del n..$^{\circ} 20$ ). - Se sopra una figura $A$ giace un altra figura $B$, si potrà muovere $A$ in modo che $B$ coincida con una figura qualunque uguale $C$.

Nota 9..$^{a}$ (al Teor. 2. del n. ${ }^{\circ} 20$ ). - Un punto di un ramo (che non sia il suo estremo) lo divide in un tratto ed in un ramo.

'T'uritio, I I novembre 1801 . 